\title{
LAS DECISIONES DE FINANCIAMIENTO: UN ANÁLISIS DESDE LA CONDUCTUALIDAD
}

\author{
FINANCING DECISIONS: AN ANALYSIS \\ BASED ON BEHAVIOR
}

\author{
Jorge Bernal Peralta ${ }^{1}$, Giomar Walter Moscoso Zegarra ${ }^{2}$ y Ernesto Leo Rossi ${ }^{3}$
}

\section{RESUMEN}

El proceso de tomar una decisión financiera, parte del supuesto de que cada una de las acciones que se planifiquen o discutan involucra dos aspectos: la maximización de utilidades y la reducción de costos. Por esto, se supone que dichas decisiones son enteramente racionales. Aquí nace la interrogante principal: ¿las personas toman decisiones racionales, no dejándose influenciar por factores conductuales que afectan la calidad de la decisión y su resultado?

Por lo anterior, se busca determinar si las decisiones financieras que se toman en las empresas familiares son racionales y por tanto responden a una jerarquía de preferencia en la utilización de recursos financieros (Pecking Order Theory), o existen factores conductuales que realmente afectan la decisión.

El estudio realizado es de diseño transeccional y de tipo relacional. Se consideró una muestra de 58 gerentes de empresas familiares del sector comercial de la región de Tacna (Perú), que contaban con una planilla superior a 20 trabajadores y por lo menos 10 años de actividad.

La investigación permitió concluir que el factor optimismo no tiene relación con las decisiones financieras, pero el factor confianza si tiene una relación media con las decisiones financieras. Se concluye, que los individuos que tienen relación con las decisiones de financiamiento de las empresas comerciales de la región, prefieren el financiamiento interno, a través del financiamiento por recursos propios, la reinversión de las utilidades retenidas y por tanto, la preferencia de capitalizarlas en lugar de repartir dividendos entre los socios.

Palabras claves: Finanzas Conductuales, Decisiones de Financiamiento, Endeudamiento, Pecking Order Theory.

Recepción: 30/08/2018. Aprobación: 29/11/2018.

\begin{abstract}
The process of making a financial decision is based on the assumption that each of the actions that are planned or discussed involved two aspects: profit maximization and cost reduction. Therefore, these decisions are assumed to be entirely rational. Here is the main question: do people make rational decisions, not being influenced by behavioral factors that affect the quality of the decision and its outcome?

Therefore, it is sought to determine if the financial decisions that are made in family businesses are rational and therefore respond to a hierarchy of preference in the use of financial resources (Pecking Order Theory), or there are behavioral factors that actually affect the decision.

The study is of transactional and of relational design. A sample of 58 managers of family businesses in the commercial sector of the region of Tacna (Peru) was considered, with a payroll of more than 20 workers and at least 10 years of activity.
\end{abstract}

1 Facultad de Administración y Economía, Universidad de Tarapacá, Arica - Chile, e-mail: jbernal@uta.cl

2 Escuela de Postgrado Neumann, Tacna - Perú, e-mail: gmoscoso@neumann.edu.pe

3 Escuela de Postgrado Neumann, Tacna - Perú, e-mail: eleo@neumann.edu.pe 
The research allowed to conclude that the optimism factor is not related to financial decisions, but the confidence factor has an average relation with the financial decisions. It is also concluded that individuals who are related to financing decisions of commercial enterprises in the region prefer internal financing, through financing by their own resources, reinvestment of retained earnings and therefore, the preference to capitalize them instead of dividing dividends between the partners.

Keywords: Pecking Order Theory, Behavioral Finance, Financing Decisions, Indebtedness.

\section{INTRODUCCIÓN}

Son tres las decisiones clásicas en las finanzas: las de inversión, que buscan maximizar la rentabilidad de los excedentes de efectivo de la empresa en el menor tiempo posible, las de dividendos que determinan el destino de las utilidades del periodo (capitalización o reparto en forma de dividendos) y las de financiamiento, que buscan conseguir siempre el menor costo de capital, la sostenibilidad en el pago y la maximización de valor de la organización.

Asimismo, la Pecking Order Theory determina que, ante la disyuntiva de la fuente de financiamiento, la preferencia es dominada por el aporte de capital accionario antes que el acudir a terceros para la obtención de dichos recursos. Es aquí donde el presente trabajo incluye variables adicionales a la decisión desde la perspectiva de las finanzas conductuales que sostiene que factores como el optimismo, el anclaje y ajuste, el enmarcado, entre otros, tienen una relación determinante en las decisiones que se toman en las empresas investigadas.

Por tanto, las conductualidad en las finanzas basan su constructo en el hecho de que las personas (y por tanto el ente ficticio: empresa) cuando sienten la presencia de la incertidumbre poseen solo un sentido abstracto de la racionalidad deseada. Por ejemplo, en los procesos de sucesión de las empresas familiares, el contexto en que se toman las decisiones puede tener influencia en la determinación final, algún escenario político o social excepcional, un proceso de fusión o escisión empresarial, un determinado clima laboral, la muerte de un familiar, entre otros, que hacen que quienes son los responsables de tomar decisiones adopten una posición diferente a lo racionalmente esperado.

Por tanto, la presente investigación busca determinar en una primera instancia si los responsables de tomar decisiones lo hacen de manera racional o están influenciados por sus propias emociones. En una siguiente fase se identifica cual es la predilección de estos sobre las estructuras de financiamiento deseadas.
Para esto, se presenta una serie de resultados que describen si las decisiones tomadas en las empresas investigadas obedece a la Pecking Order Theory que determina que las empresas privilegian el financiamiento interno antes que el externo, bajo un enfoque racional o toman decisiones influenciados por aspectos conductuales como un determinado estado emocional, manejo de paradigmas sin verificación, la confianza, el efecto de enmarcado, entre otros que están en afectando el supuesto de la racionalidad de las decisiones financieras.

Revisión de literatura. Las Finanzas tienen tres decisiones fundamentales: inversión, dividendos y financiamiento. Sobre esta última se ha teorizado y postulado modelos que intentan determinar los factores predictivos que influyen en la construcción de la estructura de capital de una empresa. Al respecto Moreira \& Rodríguez (2006) en un estudio aplicado a 74 empresas de la Bolsa de Valores Portuguesa determinaron que el endeudamiento desempeña un importante papel en la financiación del déficit ocasionado por la realización de nuevas inversiones. Asimismo, en la revisión del marco teórico expresan que, la teoría de la jerarquización financiera o teoría del Pecking Order (PO) sugiere un orden bajo el cual las personas realizan la elección de la fuente de financiamiento cuando esta afronta un déficit financiero.

Berlingeri (2003) en su estudio "Trade off o Pecking Order, una investigación sobre las decisiones de financiamiento, evalúa el grado en que las predicciones del modelo se ajustan a la realidad de las empresas. La conclusión más relevante, es que la POT explica mejor la conducta financiera de las organizaciones, pero encuentra que dicho modelo no es aplicable cuando se trata de empresas jóvenes y en crecimiento o con empresas de países emergentes. Sarmiento \& Salazar (2005), en su investigación confronta los resultados teóricos y empíricos sobre los determinantes en la construcción de la estructura de capital. A través de dicho estudio se explica que existen diferentes prácticas que se aplican 
en el momento de tomar una decisión de financiamiento de fuente menos costosa. Además, determina que, elegidas la estructura financiera, esta se convierte en óptima cuando esta permite maximizar el valor de la firma al menor costo. Esto, dentro del contexto de que, según la $\mathrm{PO}$, al respetar el mandato de los accionistas, la mejor selección es el autofinanciamiento.

En un estudio posterior, en donde se toma una muestra de 39 empresas en un periodo de tiempo de 10 años, se identifica que en concordancia con la PO las empresas más grandes están más diversificadas y por tanto presentan menores costos de asimetrías de información, lo que les genera la necesidad de un desapalancamiento del negocio. Contrasta sus resultados contra otra teoría "Static Tradeoff" que sugiere que las empresas con mayores capitales presentan menos costos de bancarrota, son menos riesgosas y por tanto la estructura de capital es influenciada por las condiciones que va presentando progresivamente el mercado (Wadnipar \& Cruz, 2008).

También aporta a la discusión, el estudio realizado por Barberis and Huang (2008) denominado "Stocks as Lotteries: The Implications of Probability Weighting for Security Prices" y concluye en que muchos fenómenos financieros son difíciles de entender en el contexto del paradigma de utilidad esperado. Por lo tanto, puede ser útil documentar las implicaciones de las teorías de utilidad inexistentes y ver si ofrecen nuevas predicciones que pueden ayudarnos a dar sentido a los mercados financieros.

Barberis \& Xiong (2010) en su estudio "Realization utility", también desarrollan sobre las decisiones que se toman en las organizaciones y determinan que los inversores nunca venden voluntariamente las acciones a pérdida, mientras que en realidad lo hacen claramente. Esto claramente describe que existen otras variables diferentes a la racionalidad absoluta que están presentes al momento de tomar una decisión.

Barberis \& Thaler (2003), aportan al estudio de la conductualidad postulando "A Survey of Behavioral Finance" en donde se analizan los límites del arbitraje, que sostiene que puede ser difícil para los decisores racionales deshacer las desviaciones causadas por entes menos racionales; y la psicológica, que aborda los factores que generan distancia sobre la racionalidad.

En su estudio Mongrut, Fuenzalida, Pezo \& Teply (2010), contrariamente a lo esperado rechaza la afirmación de la jerarquización financiera, es decir, determina que la PO no contribuye a explicar la política endeudamiento de las empresas en el periodo analizado. Dicha conclu- sión sugiere que las organizaciones tienen predilección por contraer deuda en lugar de recurrir al autofinanciamiento con los fondos generados. Este resultado contradice el modelo de jerarquización financiera que sostiene que las empresas seleccionan sus fuentes de financiamiento en función del costo de capital de estas, posteriormente recurren al autofinanciamiento y tienen como última opción la emisión de acciones.

Un estudio mucho más acotado de Ferrer \& Tresierra (2009) determina que los modelos que describen cómo se determinan las estructuras de capital se enfrentan a varias limitaciones cuando tratan de explicar el comportamiento financiero de las Pymes principalmente porque dichos supuestos teóricos sólo pueden ser verificados parcialmente en la estructura de capital finalmente adoptada. Esto, porque a diferencia de las grandes empresas, los problemas de asimetría de la información son mayor por la informalidad y la falta de historial financiero y operativo. Además, por su misma naturaleza, las Pymes generalmente poseen la motivación de mantener la propiedad y el control del negocio, condicionando con esto sus propias decisiones financieras.

Asimismo, Mondragón-Hernández (2011), en la revisión teórica describe que la PO supone que las empresas no tienen una estructura óptima de capital como si lo afirma la teoría del trade off, sino, esta depende de la disponibilidad de información si es que se tratase de mercados perfectos. También, encuentra diferencias con las investigaciones de Modigliani \& Miller por la noción de valor de mercado de la firma.

Aybar, Casino \& López. (2001) en su trabajo intenta obtener evidencia empírica acerca del cumplimiento de la PO en la definición de la estructura financiera de las empresas, diferenciando en sus hipótesis de trabajo, las Pymes y la gran empresa, teniendo como resultado que en las empresas pequeñas se cumple parcialmente dicha teoría y confirma que las oportunidades de crecimiento si influyen de manera relevante en la estructura financiera adoptada estas organizaciones. En la revisión teórica del estudio, se explica que el enfoque PO se aplica de forma específica también a las pequeñas empresas, donde las asimetrías de información son mayores. Esto, se sustenta en la frecuencia con la que dichas empresas son gestionadas por un solo director que es accionistas principal o único del capital accionario. Dicha decisión, tiene como objetivo evitar el intrusismo en la gestión, pero no necesariamente sea el fin principal conseguir una estructura de capital óptima. Por otro lado, Jiménez \& Palacin (2007), observan que las em- 
presas medianas y pequeñas tienen diferencias relevantes en su nivel de deuda, escenario que no se repite en el grupo de las grandes empresas.

Otro estudio importante, realizado por Briozzo, Vigier, Castillo, Pesce \& Speroni (2016) en el que analizan si existen características diferenciales en las pequeñas y medianas empresas en función de su tamaño y su constitución legal en empresas de la Argentina. El resultado que se obtiene es que las empresas de mayor tamaño si deciden por la obtención de deuda. Por su parte Kahneman (2015), afirma que la heurística de juicios es útil, pero a veces conduce a graves errores sistemáticos. Dicho estudio se concentra en los sesgos, ya que estos proporcionan evidencia relevante sobre la heurística de los juicios.

Sevdalis \& Harvey (2009), aportan una explicación de cómo la influencia del comportamiento y los sesgos cognoscitivos provocan una asimetría dentro de los procesos de negociación derivado de una aversión al desprendimiento de los productos por parte de los vendedores, ya que estos ignoran las posiciones de compra buscando, en un inicio, satisfacer el deseo de obtener utilidades altas y se rehúsan a una negociación para disminuir el precio de sus productos porque sienten temor al desprendimiento de los mismos a costes muy bajos, lo que afecta el comercio y el rendimiento de los negocios.

Alves (2011), comprueba la influencia de heurísticos como el comportamiento de manada, anclaje y ajuste y la contabilidad mental en los ejecutivos de distintas empresas portuguesas, afirmando que cuando mayor es el ambiente de incertidumbre los heurísticos antes mencionados tienen una mayor presencia. Asimismo, Kahneman \& Tversky (1986) en el desarrollo de su teoría prospectiva declaran que "cuando nos enfrentamos con la teoría de asignar probabilidades a resultados inciertos las personas tienen predisposición a usar las heurísticas cognitivas que tienen como producto, sesgos sistemáticos.

Conthe (2003) en su investigación describe que a pesar de que la Teoría de la Perspectiva (TP) y la psicología de las finanzas son relativamente recientes, ya se han aplicado en muchas empresas y sectores. Esto, debido a que la Teoría de la Perspectiva tiene base empírica que aspira a probar como las personas se comportan en realidad, dejando de lado muchas veces la racionalidad. El estudio de Morales y Rodríguez (2016) describe que la gestión de la innovación necesita para que se tomen las decisiones, un ambiente de incertidumbre, sin perjuicio de la calidad de la determinación. Cortada de Kohan (2008), en su estudio hace una presentación de heurísticos y sesgos de Kahneman, en cuya investigación se demuestra que, en las intuiciones de las personas sobre la probabilidad de los hechos, se producen muchos sesgos como la falacia de la ley de los pequeños números, el de conjunción, entre otros. Asimismo, Kahnemann \& Tversky (1986) también describe tres heurísticos con fines generales: accesibilidad, la representatividad y el anclaje o ajuste.

\section{METODOLOGÍA, MATERIALES Y MÉTODOS}

La presente investigación es de enfoque mixto, ya que se utilizarán datos cuantitativos y cualitativos para el cumplimiento de los objetivos de la investigación. Asimismo, es descriptivo porque expone datos sin alteración previa de los investigadores y es relacional ya que se pretende establecer si existe relación entre la conductualidad y las decisiones de financiamiento que toman los sujetos de investigación. Se opta por un diseño de tipo no experimental y a su vez transversal, ya que serán realizadas las observaciones en un momento único en el tiempo, siendo un estudio empírico y sistemático, en vista de que no se pretende manipular ningún tipo de variable.

La investigación se aplica a las empresas familiares que estén conformados como persona jurídica del giro económico comercial de la región de Tacna, Perú en el año 2016. La unidad de estudio está determinada por las empresas familiares de giro comercial, que tengan constitución como persona jurídica, tengan por lo menos 10 años de operaciones registradas y que posean una planilla superior a 20 trabajadores. Para la determinación de la muestra se aplica el muestreo no probabilístico por conveniencia. Se toman un total de 58 cuestionarios a propietarios, directivos y/o gerentes que tengan influencia directa en las decisiones de financiamiento de sus organizaciones. Para la recolección de información se utilizó como instrumento una encuesta extraída del estudio realizado por Velásquez, Tarqui \& Cáceres (2016) que es una adaptación del instrumento presentado por Cárdenas et al (2014).

\section{RESULTADOS}

Sobre el exceso de optimismo, dicha anomalía ocasiona la sobrestimación de la frecuencia y probabilidad con la que se obtendrán resultados favorables, esto influye en las decisiones financieras que toman las empresas investigadas. 
Gráfico 1. Exceso de Optimismo.

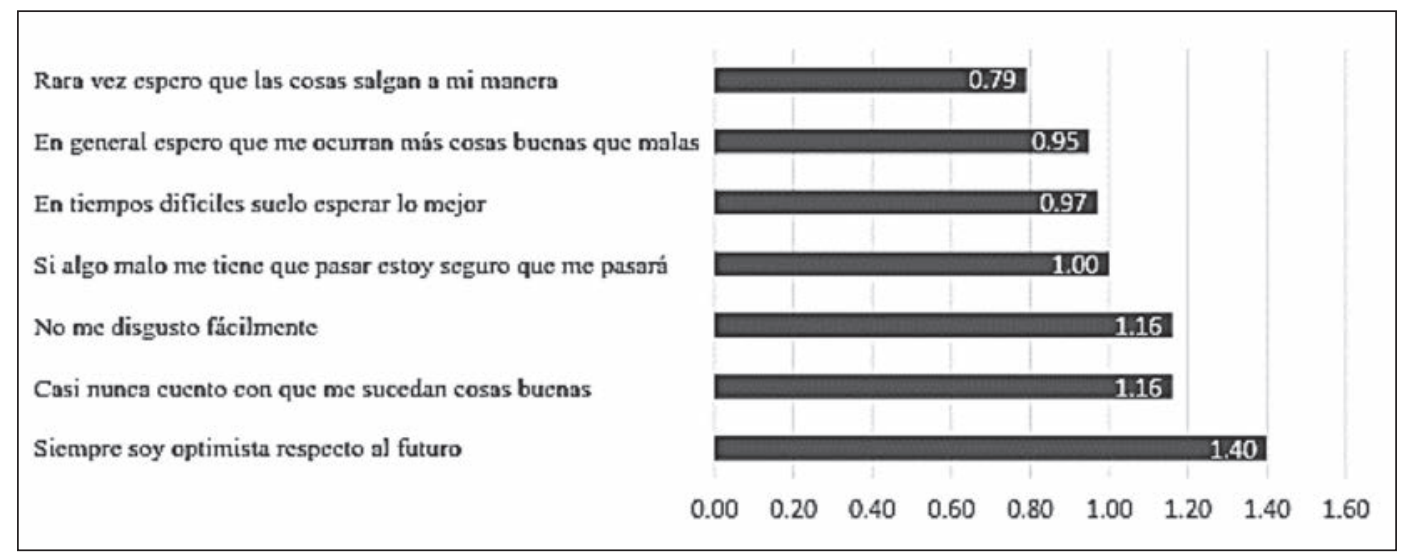

Fuente: Elaboración propia.

En base a los resultados determinados en el gráfico 1, con un promedio de 1.06 se determina un nivel de optimismo superior al promedio entre las empresas investigadas con respuestas como "Soy optimista respecto al futuro" y "Casi nunca cuento con que me sucedan cosas buenas". La fuerte presencia de dichas afirmaciones podría generar que el decisor espere sucedan hechos favorables con mayor frecuencia, alejándose mientras el indicador es más alto de la racionalidad absoluta. El "Optimismo" con un coeficiente de relación de -0.464 descarta su influencia estadística con las decisiones financieras al registrar un nivel de significancia superior al 0.05. Dicho resultado probablemente se genere por las características que se presentan en las empresas familiares ( $100 \%$ de las encuestadas) como la aversión al ingreso de capitales externos por la probabilidad de perder el control de la organización y el temor a la "desfamiliarización".

Sobre el exceso de confianza, dicha variable intenta medir las consecuencias que tiene en las decisiones de las personas en entornos donde la calidad del juicio es ambigua en el corto plazo, suelen creer que tienen mayores capacidades, habilidades y conocimientos de los que realmente poseen o que estos se encuentran por encima del promedio, alejándose de la racionalidad y acercándose a la "corazonada". Las consecuencias del exceso de confianza en el campo de las finanzas pueden estar relacionadas al sobrendeudamiento por la creencia de futuros resultados positivos de la empresa obviando la coyuntura desfavorable en la que se pueda encontrar la organización.

Gráfico 2. Exceso de Confianza.

\begin{tabular}{|l|l|l|}
\hline Inversión con ricsgo y poco tiempo & \\
Rendimientos supuestos & & \\
Es importante para mi mantenerme ocupado & & \\
Cuan buen trabajador me considero respecto a los demás \\
Disfruto bastante de mis amistades
\end{tabular}

Fuente: Elaboración propia. 
En base a los resultados del gráfico 2, se alcanza un ponderado de 0.57 , que demuestra un nivel ligeramente superior a la media de confianza. Se determina un coeficiente de relación de 0.462 y un nivel de significancia menor a 0,05 . Esto afirma que ante una menor confianza ("Puedo invertir con riesgo y poco tiempo" con -0.97) en las personas que integran las organizaciones investigadas, la estructura de financiamiento que eligen podría orientarse hacia el privilegio del recurso propio. Por el contrario, si las respuestas se acercan al puntaje ideal de 2 con las afirmaciones: "Me resulta fácil relajarme", "Disfruto de mis amistades" y "Me considero mejor trabajador que los demás" se podrían generar escenarios de sobrendeudamiento que afectarían la sostenibilidad empresarial.

Sobre la teoría prospectiva, esta explica el proceso de toma de decisiones bajo riesgo e incertidumbre. Se basa en que los individuos perciben los resultados de sus elecciones en función al impacto, en relación a un punto de referencia, que generaría en la variación de su riqueza o bienestar. Por ejemplo, intenta explicar por qué los individuos primero venden los activos que les han generado mayores retornos, pero conservan por mucho más tiempo aquellos bienes que si bien es cierto les han generado pérdidas, se espera que en algún momento puedan generar el retorno esperado, alejándose de la racionabilidad en dicha decisión.

Gráfico 3. Efecto de Prospectiva.

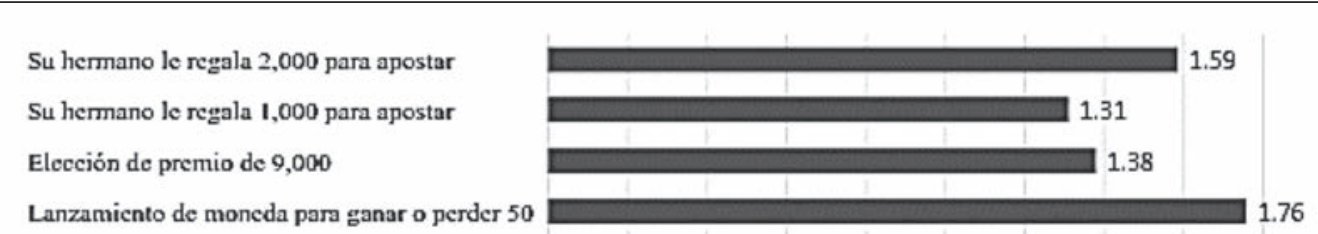

Fuente: Elaboración propia.

El gráfico 3 presenta los resultados del efecto de dicha teoría que explica como las personas toman decisiones influenciadas por la alta aversión al riesgo por considerar siempre una doble probabilidad de pérdida ante una ganancia estimada (En el lanzamiento de una moneda, usted puede perder S/ 50 o ganar S / X. ¿Cuánto debería ser X para que usted decida aceptar la apuesta? Alcanza un nivel de relación de 0.936, pero estadísticamente se determina que no tiene influencia en las decisiones de financiamiento de las empresas investigadas.

Sobre el efecto de enmarcado, refleja que un mal análisis o entendimiento de la situación, escenario o coyuntura puede distorsionar una decisión que inicialmente era absolutamente racional y convertirse en una determinación que se desvíe de lo correcto.

Los resultados presentados en el gráfico 4, registran un ponderado de 1.24 y un nivel de relación de -0.478 sugiere que, ante un análisis de escenarios menos profundo y correcto (ambos enunciados planteados de diferente forma debían generar la misma respuesta), la opción de financiamiento podría cambiar. Sin embargo, se descarta su influencia con las decisiones de financiamiento por alcanzar un nivel de significancia superior a 0,05.

Gráfico 4. Efecto de Enmarcado.

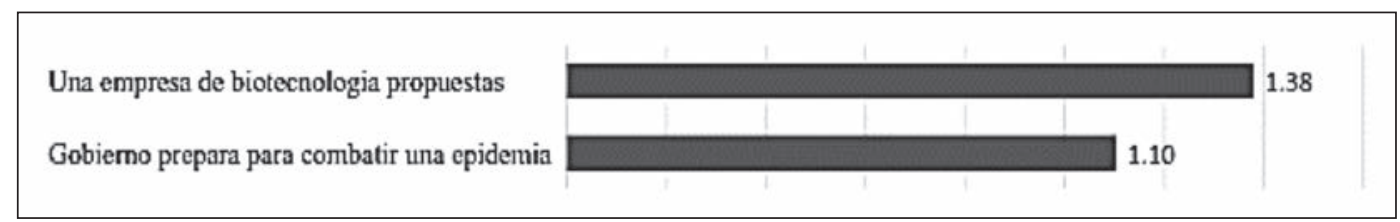

Fuente: Elaboración propia. 
Sobre el anclaje y ajuste (Tversky \& Kahneman, 1974), expresan que los individuos al efectuar estimaciones, suelen iniciar con un valor referencial que puede haber sido sugerido en la formulación del problema o con el cual están familiarizados, realizando modificaciones posteriores que les permitan llegar a la respuesta final. No obstante, estos ajustes generalmente son insuficientes, lo que genera que los resultados se encuentren sesgados hacia el punto de partida. Es decir, muchas veces al momento de tomar una decisión, partimos con información que no es confiable y por el factor de anclaje y ajuste consideramos como verdad, lo que finalmente influye en la determinación final.

Los resultados reflejan ponderados de $0.88 \mathrm{y}$ 1,71 diferentes (Tabla 1), lo que indica la fuerte presencia de dicha variable al momento de tomar una decisión que considera como una información inicial, correcta o incorrecta, puede influenciar en la determinación final por efecto de anclaje y posterior ajuste insuficiente. Se obtiene una relación de 0,478 que especifica una relación positiva media con las decisiones de financiamiento. Estadísticamente se aprueba por ser menor al nivel de significancia de 0,05.

Tabla 1. Anclaje y Ajuste.

\begin{tabular}{|l|c|}
\hline \multicolumn{1}{|c|}{ Heurístico de anclaje } & Ponderado \\
\hline Tasa de desempleo en Alemania & 0.88 \\
\hline Tasa de desempleo en España & 1.71 \\
\hline
\end{tabular}

Fuente: Elaboración propia.

Sobre el costo hundido, describe que la tendencia a considerar costos no recuperables como un factor incluyente en la decisión, puede generar perjuicios económicos provenientes del desperdicio de recursos en oportunidades inviables que podrían utilizarse para financiar negocios rentables.

En el caso del costo hundido, se obtiene un ponderado de 1.71 (Tabla 2) y un nivel de relación de 0,837 que determina un nivel de relación positiva y fuerte con las decisiones de financiamiento. Dicho resultado se generó al aplica la pregunta: "Suponga que hace 6 años usted ad- quirió un departamento al contado. Actualmente tiene la intención de venderlo, pero el precio de mercado de los departamentos ha descendido considerablemente. ¿Cuán importante sería para usted al momento de venderlo, el precio al cual adquirió dicho inmueble?"

Esto significa que se presenta una fuerte presencia de la tendencia que tienen las personas en considerar los costos irrecuperables como un factor a evaluar al tomar una decisión final al acercarse el promedio de las respuestas a la posición "Muy de acuerdo".

Tabla 2. Consolidado de la "Falacia del costo hundido".

\begin{tabular}{|l|l|}
\hline Falacia del costo hundido & Ponderado \\
\hline Costo en la adquisición del departamento & 1.71 \\
\hline
\end{tabular}

Fuente: Elaboración propia.

Sobre las decisiones de financiamiento, la preferencia de quienes toman decisiones en las empresas investigadas es que una vez que determina el impuesto anual a la renta y se determinan las utilidades definitivas, se decide por ingresar dichas ganancias al patrimonio empresarial con fines de inversión 1,22 (Gráfico 5).
Además, se expresa con un ponderado de 1,21 su preferencia clara por el financiamiento interno. Dichos resultados se relacionan con el anhelo de las empresas (principalmente familiares) a no renunciar al "poder total" dentro de sus empresas, sacrificando un crecimiento más estructurado y de mayores oportunidades en otros mercados. 
Gráfico 5. Decisiones de Financiamiento.

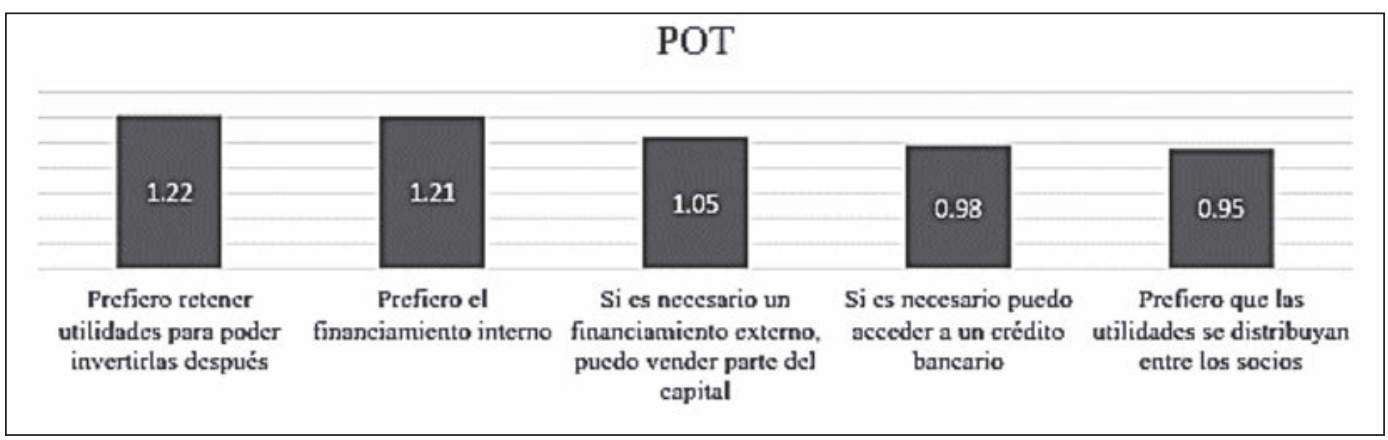

Fuente: Elaboración propia.

En relación entre la conductualidad y las decisiones financieras se establece un nivel de relación media 0.412 (Tabla 3). Esto indica que, algunas variables conductuales como la confianza, el efecto de enmarcado, el anclaje y ajuste y el costo hundido, pueden inducir a las personas a construir estructuras de financiamiento diferentes a las que elegirían en un escenario absolutamen- te relacional y por tanto, puede que finalmente no se ajusten a las necesidades de sus empresas. Dichas determinaciones pueden en un escenario negativo, generar estructuras muy apalancadas que pueden afectar la sostenibilidad de cualquier negocio o se puede registrar la ausencia de capitales externos que contribuyan al desarrollo de la empresa.

Tabla 3. Relación entre la conductualidad y las decisiones financieras.

\begin{tabular}{|ll|c|c|}
\hline & & POT & F conductuales \\
\hline POT & Correlación de Pearson & 1 &, 412 \\
& Sig. (bilateral) & &, 038 \\
& N & 58 & 58 \\
F. conductuales & Correlación de Pearson &, 412 & 1 \\
& Sig. (bilateral) &, 038 & 58 \\
& N & 58 & \\
\hline
\end{tabular}

Fuente: Elaboración propia.

\section{DISCUSIÓN Y CONCLUSIONES}

En el caso de las empresas investigadas, se parte con la premisa de que las decisiones que estas toman, parten del supuesto de la racionalidad absoluta, pero en el momento de la deliberación final también intervienen factores no planificados como el exceso de confianza, el anclaje y ajuste, el costo hundido, entre otros. Dichos factores, pueden inducir a los decisores a construir estructuras de financiamiento que no se ajusten a las necesidades de sus empresas.
Por tanto, dichas determinaciones pueden concluir en una empresa demasiado apalancada o en el otro extremo, la decisión de prescindir de fondos externos, renunciando con esto a los beneficios tributarios y a una mejor capacidad de crecimiento en el futuro.

Se concluye que, los individuos que tienen relación con las decisiones de financiamiento de las empresas comerciales de la región, prefieren el financiamiento interno, a través del financiamiento por recursos propios, la reinversión de las utilidades retenidas y por tanto la preferencia 
de capitalizarlas en lugar de repartir dividendos entre los socios.

Se establece que en las empresas comerciales de la región existe una influencia de la conductualidad en la toma de decisiones a nivel medio (correlación 0,412). Esto probablemente por la fortaleza de la POT, que privilegia el conservadurismo del empresario local al no permitir el financiamiento externo.

\section{REFERENCIAS BIBLIOGRÁFICAS}

Alves (2011). Behavioural influences in portuguese foreign direct investment. The journal of Socio-Economics, 40, 394-403.

Aybar, C., Cacino, A. \& López, J. (2001). Jerarquía de preferencias y estrategia empresarial en la determinación de la estructura de capital de la Pyme: Un enfoque con datos de panel. Documento de Trabajo del Instituto Valenciano de Investigaciones Económicas, 1(1), 5-16.

Barberis, N., Huang M. (2008). Stocks as Lotteries: The Implications of Probability Weighting for Security Prices. American Economic Review, 98:5, 2066-2100.

Barberis, N., Thaler R. (2003). A Survey of Behavioral Finance. Handbook of the Economics of Finance, 1055-1114.

Barberis, N., Xiong W. (2012). Realization Utility. Journal of Financial Economics, 251-271.

Berlingeri, H. (2003). ¿Trade off o Pecking Order? Una investigación sobre las decisiones de financiamiento. Buenos Aires: Pontificia Universidad Católica.

Briozzo, A., Vigier, H., Castillo, N. Pesce, G. \& Speroni, M. (2016). Decisiones de financiamiento en pymes ¿existen diferencias en función del tamaño y la forma legal? Journal of Management and Economics for Iberoamerica, 32(138), 71-81.

Cárdenas, C., Linares, V. \& Ruiz J. (2014) Análisis de la Estructura de Capital de Empresas No Financieras en el Mercado de Valores Peruano: Una Aproximación Conductual. Universidad Peruana de Ciencias Aplicadas, 88-97.

Cortada de Kohan, N. (2008). Los sesgos cognitivos en la toma de decisiones. International Journal of Psychological Research, 1(1), 68-73.
Conthe, M. (2003). Psicología de las finanzas. Encuentros multidisciplinares, 5(15), 55-61.

Ferrer, M. \& Tresierra, A. (2009). Las Pymes y las teorías modernas sobre estructura de capital, Compendium, 12(22), 65-77.

Jiménez, F. \& Palacín, M. (2006). Determinantes de la estructura financiera de la empresa. Revista Europea de Dirección y Economía de la Empresa, $16(4), 9-24$.

Kahneman, D. (2015). Pensar Rápido, Pensar Despacio. Barcelona: Debate.

Kahneman, D. y Tversky, A. (1986). Rational Choice and the Framing of Decisions. Journal of Business, 59(4), 251-278.

Mondragón-Hernández, S. (2011). Marco conceptual de las teorías de la irrelevancia, del trade-off y de la jerarquía de las preferencias. Cuadernos de Contabilidad, 12(30), 165-178.

Mongrut, S., Fuenzalida, D., Pezo, G. \& Teply, Z. (2010). Explorando teorías de estructura de capital en Latinoamérica. Cuaderno de Administración Bogotá, 23(41), 163-184.

Morales, C. \& Rodríguez, G. (2016). Heurísticas y Sesgos Cognitivos en la Gestión de la Innovación. Recuperado de http://service.udes.edu.co/ eisi/memorias/ ponencias/ep7.pdf

Moreira Da Silva, C. \& Rodríguez, J. (2006). Contraste de la Teoría del Pecking Order versus la Teoría del Trade-Off para una Muestra de Empresas Portuguesas. "Nuevas Tendencias en Dirección de Empresas". Recuperado: http://www.eco.uva. es/empresa/uploads/dt_01_06.pdf

Sarmiento, R. \& Salazar, M. (2005). La estructura de financiamiento de las empresas: una evidencia teórica y econométrica para Colombia (1997-2004). Bogotá: Pontificia Universidad Javeriana.

Sevdalis, N. \& Harvey, N. (2009). Reducing the impact bias in judgments of post-decisional affect: distraction or task interference? Judgment and Decision Making, 4(1), 287-296.

Velásquez, L., Tarqui, L. \& Cáceres, R. (2016). Las finanzas conductuales y su relación con las decisiones de financiamiento de las empresas familiares del sector comercial de la provincia de Tacna. (Tesis inédita 
de maestría). Neumann Escuela de Postgrado, Tacna, Perú.

Wadnipar, S. \& Cruz, J (2008) Determinación de la estructura de capital de las empresas colombianas. Revista Soluciones de Posgrado EIA, 1(1), 23-43. 